\title{
Homestead Creator: Using Card Sorting in Search for Culture-Aware Categorizations of Interface Objects
}

\author{
Kasper Rodil ${ }^{1}$, Matthias Rehm ${ }^{1}$, and Heike Winschiers-Theophilus ${ }^{2}$ \\ ${ }^{1}$ Dept. Architecture, Design and Media Technology, Aalborg University, Denmark \\ ${ }^{2}$ Dept. Software Eng. Polytechnic of Namibia, Namibia \\ kr@create.aau.dk, matthias@create.aau.dk, \\ hwinschiers@polytechnic.edu.na
}

\begin{abstract}
Designing intuitive interfaces for rural African users requires us to understand the users' conceptual model. We acknowledge differences in categorization approaches based on cultural factors, among others. In the absence of comprehensive literature and theories, we explore card sorting as a means to derive a local categorization of interface objects for one of our prototypes. Results indicate a locational-relational categorization scheme among Herero elders in Namibia.
\end{abstract}

Keywords: categorization, indigenous knowledge, 3D visualization, card sorting, $\mathrm{HCI}$, interface design.

\section{$1 \quad$ Introduction}

Since late 2010 we have investigated 3D graphics as means of contextualizing indigenous knowledge in digital representations. Besides the numerous challenges of technical constraints and understudied implications of interactions, perception and recognition by African rural dwellers we have had a major breakthrough in terms of dialogical design communication. The graphical representations have triggered uncountable discussions, narrations and design suggestions in the community. Thus at this point we further explore visual communication as a form of design dialogue to overcome language and cultural barriers. Kostelnick describes visual communication design as a continuum between global and culture-focused design, where hybridity of the two ends can exist [1]. He argues that the former 'view' is a product of cognitive perception having universal empirically testable characteristics. The latter is an argument also carried in linguistics that culture is an influencing factor. That the characteristics of visual communication are experiential and socially constructed only fully transferred within their contexts of origin. It is the balancing act between sensitivity to locally negotiated methods and artifacts of visual communication and introduction to technology that currently occupy our research and must be understood from this viewpoint. The notion we wish to raise is not to argue one or the other, but argue for the sensitivity and hybridity of these two ends. That nothing can be taken for granted - especially not appraisal of universal metaphors in interface design or knowledge management structures. 
Since the conception of this project, elders from the pilot community in Namibia have been co-designing prototypes with external designers. The elders are wellrespected and knowledgeable males, who have acquired much confidence in the usage of novel technologies as well as the ability to critically evaluate and suggest design improvements.

\section{The HomeStead Creator}

In 2011 we developed a prototype termed the HomeSteadCreator (HSC), which was received with enthusiasm by the Herero community members [2]. The HSC is running on an Android powered tablet. The village elders consider the tablet as being less intimidating and the touch interactions to be more intuitive than the usage of laptops and prototypes we have evaluated earlier.

HSC is a tool for rural Herero community members to re-create their own environment or any imaginary context with familiar 3D objects. These re-created scenarios are then combined with locally recorded IK videos and audio files to provide a digital context. Fig. 1 shows an example of the 3D re-construction of a homestead from two different cameras.

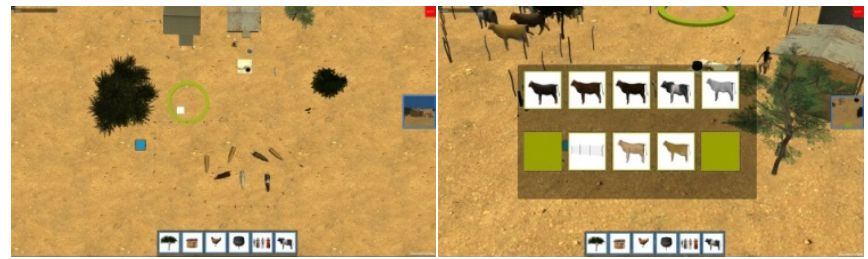

(a)

(b)

Fig. 1. A re-created homestead seen from (a) 90-degree tilt or (b) free-roam camera perspective. (a) shows the six categories as default. (b) shows the cattle category expanded.

When the HSC is launched, a textured terrain in a 3D environment seen from a 90degree tilt perspective (perpendicular to the terrain) is displayed.

The user of the HSC is presented with a row of icons representing categories defined by us. When they are being activated by a single-touch they expand and show virtual 3D objects within the 'category' (visible on Fig. 1(b)).

The objects are selected by single-touch and subsequently they are instantiated in the center of the terrain. The user can then position and rotate the object by common finger gestures (two-finger drag, two-finger circular motion etc.). The prototype was refined in 2012 based on the co-designers suggestions. From the introduction of the HSC, the tool has enhanced the design dialogue substantially while promoting crosscultural learning. E.g. the elders have shown us the correct position of the holy fire and the elder's house or 'the courtesy route around the homestead to greet the man of the house' by visualizing it on the HSC. Thus it has shown to be able to facilitate dialogue on specific issues of interaction design and as an ethnographical tool using 
visualization as a communication tool. In subsequent sessions the HSC was even expanded to be a storytelling device between elders and youths.

\section{Current Categorization Attempts}

One of the focal points in the 2012 revision was the organization of the interface with the categorization of virtual objects. This grew out of the continuous demand for new graphical objects by the elders. The 7-10 inch tablet screen can only display so many objects at a time. The current prototype has six categories with up to ten objects (see figure 2). Upon suggestions by the elders, we did a number of rearrangements of objects. In 2011, we had pre-grouped the objects according to our own categorization scheme. During the usability evaluation session of the prototype, we also explored the appropriateness of the categorization as well as the completeness of objects represented. For example the 'fence' which was previously grouped under 'objects' was requested to be moved to the 'cattle' category. The cattle were previously grouped with the other animals yet in the Herero tradition, cattle are attributed a different status than other animals thus the request for a separate category. Furthermore, the 'fire place' was requested to be part of the 'homestead items' together with the pots and washing line. See Table 1 for the updated categories.

Table 1. The figure shows the six groups with corresponding icons and objects within each group

\begin{tabular}{|c|c|c|c|}
\hline group & objects & Count \\
\hline local flora & $\begin{array}{c}|c| \\
\text { Tree }\end{array}$ & 8 \\
\hline $\begin{array}{c}\text { larger struc- } \\
\text { tures }\end{array}$ & House & $\begin{array}{c}\text { houses, large solar panel, } \\
\text { water pump, water tank }\end{array}$ & 9 \\
\hline $\begin{array}{c}\text { smaller an- } \\
\text { imals }\end{array}$ & Chicken & $\begin{array}{c}\text { goats, sheep, dogs, chicken } \\
\text { objects }\end{array}$ & 7 \\
\hline people & $\begin{array}{c}\text { cooking pot, table, washing } \\
\text { Cooking fireplace, chair }\end{array}$ & 5 \\
\hline cattle & People & $\begin{array}{c}\text { people in various age } \\
\text { groups, attires and postures }\end{array}$ & 10 \\
\hline
\end{tabular}

These seemingly minor re-arrangements reminded us of the underlying differences of conceptualization and categorization between the co-designers in the village and us.

We acknowledge that with the increasing number of objects, neither scrollable lists nor our pre-ordered categorization system is adequate. Instead of continuously reiterating the categorization of newly added objects we attempt to conceptualize the underlying structuring of objects.

\section{Situated Concepts in a Local Ontology}

From a practical technology design point of view, we need a guiding ontology that captures the concepts and relations between them for a re-contextualization of the 
Herero homestead. Defining an ontological representation will allow for sharing and re-using the captured knowledge [3]. Assuming a culture-specific categorization of the domain, the ontology will not only be useful for the obvious task of structuring menu items in the HSC. It will also capture parts of the intangible knowledge of the user group [4], thus allowing to create knowledge sharing applications that can partially construct relevant scenarios automatically based on the encoded knowledge.

Rehm [5] has focused on a situated acquisition of concepts and has shown the usefulness of combining nature and nurture views as described by Rosser [6]. On the one hand he has taken into account information processing and learning routines that have developed over time and are shared by all humans (nature perspective). On the other hand, based on these processing abilities, categorizations and concepts are developed by individual experiences, thus shaping an individual conceptual system that is in accordance with one's experiential history, taking into account environmental as well as social factors (nurture perspective). For the sake of the task we are interested in defining the relevant categories and their relations in the domain of the HSC. The 'fences and cows' example highlights the fact that there is no 'universal' conceptualization. Instead, we have taken into account Lakoff's ideas of situated concepts that cannot be viewed independent of the context of their use [7]. And as Ingold explains: "...to individuals who belong to different intentional worlds, the same objects in the same physical surroundings may mean quite different things. And when people act towards these objects, or with them in mind, their actions respond to the ways they are already appropriated, categorized or valorised in terms of a particular, pre-existent design." [8].

Not being members of the Herero culture we lack crucial information about the use of objects rendering the categorizations that we create from our own experiential history useless in the given cultural context. Studies have investigated these cultural differences. For instance Nisbett et al. found a difference between being either holistic (East-Asians) and analytical (Westerners) [9]. Hunn describes how the Tzeltal peoples (a Mayan ethnic group) classify butterflies and moths [10]. They distinguish butterfly larvae into sixteen terminal groups due to characteristics of being edible, dangerous to crops etc. The adult butterflies do not have these characteristics (although being visually distinguishable) thus they are (locally) not important for categorization. Similarly, do members of the Herero community distinguish cattle from other animals and require a larger variety of the same type. Local trees are by the elders also not necessarily classified by species, but by characteristics that make them appropriate for the homestead or the kraal. To determine a local categorization scheme of virtual objects we have turned to card sorting as a means to find a local ontology for further developing the object structures in the HSC [11].

\section{$5 \quad$ Card Sorting as a Method for Establishing the Local Classification Scheme}

Card sorting is a traditional HCI method to organize information in web pages into meaningful categories and for intuitive retrieval of information. 
The method is usually either an 'open' (generative type) or 'closed' (evaluative). One of the benefits with the 'open' version is that participants can categorize objects without pre-defined sorting, themes or adhering to an overlying taxonomy. Conversely, the 'closed' version is suitable for establishing at what level participants agree with the categorization and terms used for pre-sorted groups.

Card sorting is valuable for gaining insights into the participants' mental models by eliciting how they sort, group and organize items. Petrie el al. showed that card sorting can illuminate cultural differences in the mental models behind information architectures [12]. The product of the card sorting method is a snapshot of a subjective categorization and developers might be fixed on the final sort to implement information architecture from. However we argue that the method itself (especially the open version) is also effective in creating a dialogue around the user's standpoint. Thus in the process of sorting the researcher gains an insight on the users' world views.

\section{Method}

In total we conducted the study at three different Herero sites in Namibia. With a total number of participants being 5 females and 9 males. The presentation of detailed results is beyond the scope of this paper, therefore we will only present results from the 5 elders from our long-term collaboration village.

In preparation for the card sorting sessions a set of 47 cards was printed. The set consists of laminated cards with images of the 3D objects from the HSC, which are rendered with a white background. The cards are printed as they appear as icons in the six interface categories in the HSC.

The village elders who are the future users of the HSC and have been co-designers on the project from its conception were recruited for the sessions. The sessions were decided to be facilitated individually to investigate each elder's viewpoint. The elders were prompted to say what they saw on the cards and to talk aloud while sorting, this was to confirm recognition of the represented $3 \mathrm{D}$ objects and to understand the rationale for the sorting decisions.

The agenda was explained before the sessions began and we stressed the openness of the studies by emphasizing that nothing was regarded wrong or right and their help would aid in improving the prototype. An open card sorting was chosen for not to impose any overlaying structure potentially overriding the local way of categorization. Thus the elders were instructed to group the cards as they preferred.

The sessions were documented with video recordings, still photographs, observation and interview notes. In the case of a participant not being English speaking the interviews were facilitated by a local Herero co-researcher. An independent Herero translator translated the videos after the field trips to minimize translation bias.

\section{$7 \quad$ Results}

We were able to recruit 5 elders in the village. Fig. 2 displays the 5 final card sorts from the study conducted in the village. 
The first participant (Vehiha) began by looking at all the cards he was given. After about five minutes, he began placing cards into five, laid out, distinguishable groups with all the cards facing up. He said that the two groups representing the homestead could be piled together since they represent the same. The cards were completely mixed when relating the now four card groups (see Fig. 2 (a)) to the six interface categories we implemented in the HSC. It puzzled us that he had actually made a map of the village. The four groups were each representing a place within the village. Marked on Fig. 2 as: 1: [homestead(s)], 2: [group of goats], 3:[cattle kraal] 4: [community water pump area].

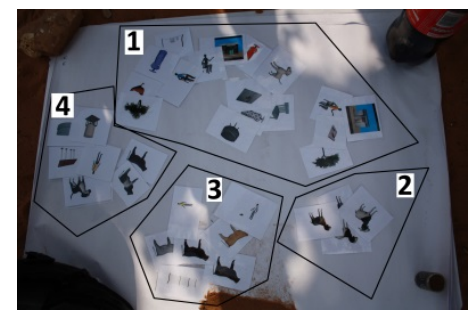

(a) Vehiha

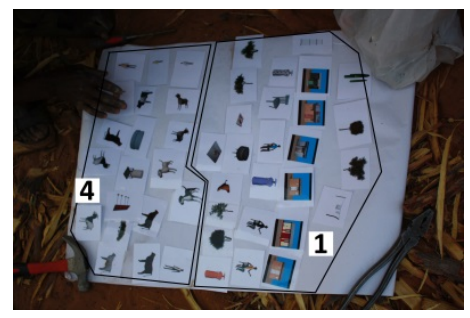

(b) Job

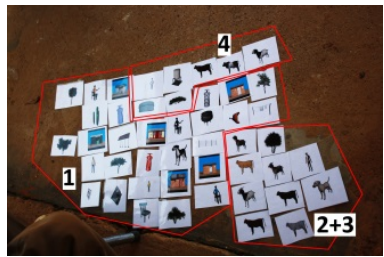

(c) Gerrard

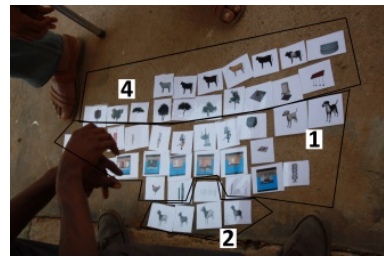

(d) Beau

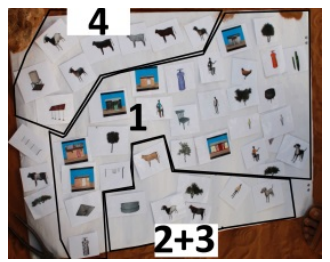

(e) Benji

Fig. 2. The pictures show the final card sorts by the 5 village elders. Annotations are added to visualize the categorization of cards based on a location-relational categorization scheme.

For instance, one card group contained cards depicting the community water pump (where they bring their cattle for water), a young boy, a bull, a dog, a cow, a solar panel and a water tank. He said that there would always be a young man taking care of the cattle and being responsible for herding them between the kraal (animal enclosure) and the water pump. Thus, the young man belonged to that water pump group. Then Vehiha added a boy to the cattle group (3) and said (trans.): "while the big man is busy at the homestead the others are at the kraal." So the 'boy' card belongs there too.

We asked where we should place a certain tree. He said that specific trees in the bush do not belong in the groups he sorted, but there should be a tree for the boy to rest under at the water pump group, which he then added. The other elders followed the same categorization scheme, and where consistent each in making a group for the homesteads (1) and the water pump (4). Participant 2 (Job) emphasized the layout of the homestead and the order of objects relational to each other situated there. For instance by showing that the man of the homestead per tradition sits to the right of the 
entrance between the house and the holy fire (see Fig. 2 (b)). This explanation was consistent with similar explanations given by the elders, thus the card sorting method was also a trigger for sharing local customs.

All cards were placed according to location of where the real objects/people represented as cards are functioning or located in relation to each other. Unsurprisingly none adhered to our categorization scheme. It must be stated that all findings across the three sites were showing the same categorization scheme as the results as from the five elders reported here. And for instance when we prompted on where to place the snake (this was a hypothetical card mentioned in the discussion), the participants stated that the snake would not belong in any group, since snakes have no purpose within the village.

\section{Discussion}

The open card sorting proved very useful since it was possible to uncover an unexpected categorization scheme. The exploratory nature allowed external researchers to ask follow-up questions ensuring that the rationale behind the categorization was transferred from the participants. It also accommodated through dialogue, that the categorization would need duplicates of some objects per group. This finding is highly interesting since card sorting is often used to categorize an item only one place based on participants' suggestions. Here it was established -contrary to our categorization scheme- that some objects should be multiple places based on participants' suggestions. The set of cards did not have duplicates for all objects, which actually shows how our method was influencing the study with a preconception of dichotomous thinking. For instance if there were more 'fence' cards the participants would have been able to separate the groups more visibly as the places are separated by fences in the village. Conversely, the elders were able to place more than one 'boy' card since we made three 3D models of a boy (thus more cards). Although we gained the feedback in the sessions on this matter, further studies should have more cards of the same type to accommodate the possibility to place many of the same type.

\section{Conclusion}

All findings from the categorization tasks point towards a shared local way of grouping the cards. We observed a 'place' based pattern, where objects meaningful to a location are grouped together, such as the 'boy' that looks after the 'cattle' at the 'kraal'. The open card sorting method supported the discovery of a new categorization scheme which we call location-relational. The pattern confirms prior research on Herero's prioritization of place as a meaningful location with activities and protocols attached to [13]. The findings dramatically changes our previous design ideas and future retrieval of interface objects will follow this new categorization scheme for ensuring intuitiveness and preservation of tacit knowledge in the interface.

The full data set is sufficient for the purpose of informing our local design since it is in the hands of this study's elders the HSC will be used. We proceed to refine the 
study for robustness, investigate the many details left out for this contribution and conduct follow-up studies. We strongly encourage researchers to pursue the data collection and analysis for a sound theory building in this under-researched area.

\section{References}

1. Kostelnick, C.: Cultural adaptation and information design: two contrasting views. IEEE Transactions on Professional Communication 38(4), 182-196 (1995)

2. Rodil, K., Winschiers-Theophilus, H., Jensen, K.L., Rehm, M.: Homestead creator: a tool for indigenous designers. In: Proceedings of the 7th Nordic Conference on HumanComputer Interaction: Making Sense Through Design, NordiCHI 2012, pp. 627-630. ACM, New York (2012)

3. Gruber, T.R.: Toward principles for the design of ontologies used for knowledge sharing. International Journal of Human Computer Studies 43(5), 907-928 (1995)

4. Unesco: The UNESCO Convention for the Safeguarding of the Intangible Cultural Heritage, http://www. unesco.org/culture/ich/en/convention/ (accessed May 6, 2013)

5. Rehm, M.: Being seaward-handed: a computational model of the acquisition of languagespecific spatial references. Cognitive Processing 5(1), 15-30 (2004)

6. Rosser, R.A.: Cognitive development: Psychological and biological perspectives. Allyn and Bacon Boston (1994)

7. Lakoff, G.: Cognitive models and prototype theory. In: Neisser, U. (ed.) Concepts and Conceptual Development: Ecological and Intellectual Factors in Categorization, vol. 1. Cambridge University Press (1989)

8. Ingold, T.: The Perception of Environment. Essays on Dwelling, Livelihood and Skill. Routledge, London (2000)

9. Nisbett, R.E., Peng, K., Choi, I., Norenzayan, A.: Culture and systems of thought: holistic versus analytic cognition. . Psychological Review 108(2), 291 (2001)

10. Hunn, E.: The utilitarian factor in folk biological classification. American Anthropologist 84(4) (1982)

11. Ross, J.: Comparing user research methods for information architecture (2011), http: / / www . uxmatters. com/mt/archives / 2011/06/comparing-userresearch-methods-for-information-architecture.php (accessed March 24, 2013)

12. Petrie, H., Power, C., Cairns, P., Seneler, C.: Using card sorts for understanding website information architectures: Technological, methodological and cultural issues. In: Campos, P., Graham, N., Jorge, J., Nunes, N., Palanque, P., Winckler, M. (eds.) INTERACT 2011, Part IV. LNCS, vol. 6949, pp. 309-322. Springer, Heidelberg (2011)

13. Bidwell, N.J., Winschiers-Theophilus, H., Kapuire, G.K., Rehm, M.: Pushing personhood into place: Situating media in rural knowledge in africa. International Journal of HumanComputer Studies 69(10), 618-631 (2011) 